\title{
Sleep quality, timing, and psychological difficulties in Italian school-age children and their mothers during COVID-19 lockdown
}

\author{
Cellini Nicola ${ }^{1,2,3,4^{*}}$, Di Giorgio Elisa ${ }^{5}$, Mioni Giovanna $^{1}$, Di Riso Daniela ${ }^{5}$ \\ ${ }^{1}$ Department of General Psychology \\ ${ }^{2}$ Department of Biomedical Sciences \\ ${ }^{3}$ Padova Neuroscience Center \\ ${ }^{4}$ Human Inspired Technology Center \\ ${ }^{5}$ Department of Developmental Psychology and Socialization \\ University of Padova, Padova, Italy \\ RUNNING HEAD: Sleep in children during home confinement
}

\author{
Correspondence to: \\ Nicola Cellini, Ph.D. \\ Department of General Psychology, University of Padova \\ Via Venezia 8, 35131, Padova, Italy \\ E-mail: nicola.cellini@unipd.it
}

Acknowledgments: This work was carried out within the scope of the project "use-inspired basic research", for which the Department of General Psychology of the University of Padova has been recognized as "Dipartimento di Eccellenza" by the Ministry of University and Research.

Disclosure: There are no financial or other relationships that could be interpreted as a conflict of interest affecting this manuscript. This research received no specific grant from any funding agency, commercial or not-for-profit sectors. 


\begin{abstract}
Background. By March $10^{\text {th }}, 2020$, the Italian Government has ordered a national lockdown to limit viral transmission of COVID-19 infection, which establishes home confinement, movement restriction, home (smart) working, and temporary closure of non-essential businesses and schools of every order and degree. The current study investigated how these restrictive measures impacted sleep quality, timing, and psychological difficulties in school-age children and their mothers during the lockdown.
\end{abstract}

Methods. Using an online survey, 299 mothers reported their sleep habits (timing, quality, quantity), time experience, and psychological difficulties (emotional and behavioral) and of those of their children (6-10 yrs old) during the home confinement and, retrospectively, before the lockdown.

Results. During the lockdown, children showed a marked delay in sleep timing, i.e., later bedtime and wake time, and a mild worsening in sleep quality. They were less prone to respect the daily routines and to keep track of the passage of time. We also observe increased emotional, conduct, and hyperactive symptoms, and the increase in these psychological difficulties was predicted by the change in sleep quality, boredom, and mothers' psychological difficulties. Also, mothers showed a delayed sleep timing and worsening of sleep quality during the lockdown, which degree varied depending on their working conditions. Mothers who kept working regularly outside their home during the lockdown reported a more regular sleep pattern, whereas mothers who stopped working showed more emotional symptoms and relevant changes in their time perception.

Conclusions. Overall, given the evidence of the adverse behavioral and psychological impact of home confinement and social restrictions, effective measures are needed to be in place to mitigate their long-term effects on children and their mothers, especially those in the smart working condition.

Keywords: COVID-19; children; home confinement; sleep quality, work condition

\title{
Key points and relevance:
}

- Sleep is a crucial joining link between physical and psychological wellbeing, especially for younger individuals.

- This study investigated the impact of COVID-19 prolonged home confinement in mothers and their school-age children's sleep in terms of timing and quality, and the interplay with psychological difficulties.

- Children showed a marked shift in sleep timing, i.e., later bedtime and wake time, and increased psychological difficulties, which were predicted by the worsening in sleep quality.

- Effective measures are needed to be in place to mitigate the long-term effects of home confinement in children and their mothers. 


\section{Introduction}

On March the $11^{\text {th }}$, the World Health Organization (WHO) declared the novel coronavirus (COVID-19) outbreak a pandemic. In a more striking way than in other countries, by March $10^{\text {th }}$, 2020, the Italian Government has ordered a national lockdown to limit viral transmission of COVID-19 infection, which establishes home confinement, movement restriction, home (smart) working, and temporary closure of non-essential businesses and schools of every order and degree. Although necessary, the prolonged home confinement might have been detrimental for mental health, affecting people's lives by influencing their everyday behaviour, in line with previous studies on quarantine situations (e.g., Sprang \& Silman, 2013). Most people experienced unprecedented stressful conditions that have increased psychological symptoms such as emotional dysregulation, depression, stress and anxiety (Brooks et al., 2020) but also affected their sleep quality and the circadian rhythms (Blume, Schmidt, \& Cajochen, 2020; Cellini, Canale, Mioni, \& Costa, 2020; Wright et al., 2020).

The link between sleep disturbances and behavioural (e.g., self-regulation, conduct, and attention) and emotional (depression, anxiety, and stress) difficulties has been well-established in adults, in adolescents, and even in children (Baum et al., 2014; Gregory \& O'Connor, 2002; Paavonen, Porkka-Heiskanen, \& Lahikainen, 2009). A few studies already explored the interplay between sleep habits and psychosocial well-being during the Covid-19 outbreak in large samples of Italian adults (Casagrande, Favieri, Tambelli, \& Forte, 2020; Cellini et al., 2020; Gualano, Lo Moro, Voglino, Bert F, \& Siliquini, 2020). During the home confinement period, individuals reported marked changes in sleep habits, i.e. later bed and wake time, increase time spent in bed, and poorer sleep quality. The unhealthy sleep pattern seemed to be associated with more intense symptoms of depression and anxiety (Cellini et al., 2020). Casagrande and colleagues (2020) indicated that females and people who reported fear to be infected by Covid-19 are more prone to sleep disturbances and to feel distressed. Moreover, poorer sleep quality seemed to be associated also to post-traumatic stress disorder symptoms. 
Although children are less likely to be severely infected by the virus (Kelvin \& Halperin, 2020), COVID-19 pandemic might have a dramatic impact on younger individuals due to the disruption of daily routines (Brazendale et al., 2017; Jiao et al., 2020). They experience fears, uncertainties, physical and social isolation, and may miss school for a prolonged period. The literature highlighted that even predictable school closures, such as holidays or weekends, might affect children's well-being in terms of unhealthy sleep patterns and poorer physical activity (Wang et al., 2020). The pandemic outbreak, imposing a prolonged and unexpected interruption of the normal school routine, daily activities, and connections with peers, could represent an important risk factor for physical and mental health in children and families. Sleep seemed to be a crucial joining link between physical and psychological wellbeing, especially for younger individuals (Becker \& Gregory, 2020; Carskadon \& Barker, 2020; Gregory \& O'Connor, 2002). For instance, sleep duration in school-age children is negatively correlated with behavior and mood problems (Paavonen et al., 2009) and both sleep and timing modulate the children's abilities to adequately regulate emotions (Carskadon \& Barker, 2020). Prolonged home confinement due to pandemic outbreaks might induce children to significantly postpone sleep and wake time, with an elongation of time spent in bed doing daily activities such as remote learning, studying, or playing (Guan et al., 2020). Moreover, children with irregular sleep patterns seemed to be more distressed, hyperactive, inattentive, and with poorer frustration tolerance (Jiao et al., 2020). Therefore, it is not surprising that the European Academy of CBT-I, in their guidelines for dealing with sleep problems during home confinement (Altena et al., 2020), proposed specific recommendations for mothers and their children, which are considered at high risk to develop sleep and mental problems during this unprecedented situation.

Although the literature on the effects of COVID-19 related restrictions on sleep habits in adults it is increasing rapidly (Beck et al., 2020; Blume et al., 2020; Casagrande et al., 2020; Cellini et al., 2020; Gualano, et al., 2020; Kokou-Kpolou, Megalakaki, Laimou, \& Kousouri, 2020; Lin et al., 2020; Voitsidis et al., 2020; Wright, et al., 2020), studies focusing on sleep disturbances in 
children during this pandemic are very scarce (Di Giorgio, Di Riso, Mioni, \& Cellini, 2020), targeting specific samples (i.e., children with obesity; Pietrobelli et al., 2020) or focusing on wide age ranges (i.e., 3-18 years old; Orgilés, Morales, Delvecchio, Mazzeschi, \& Espada, 2020).

To fill this gap in the literature, the present study aimed to investigate how the prolonged home confinement during the national lockdown impacted mothers and their school-age children's sleep in terms of timing and quality and to describe the interplay between children sleep and routines changes in predicting their emotional symptoms and difficulties. We expected to observe a shift in sleep timing (delayed bed and waketime), a general decrement in sleep quality in children, and a positive association between sleep quality worsening and increase emotional symptoms and difficulties. We also aimed to assess the sleep timing and quality in children's mothers and to explore how mothers and children's sleep and behavioral changes during the lockdown interacted during this period in prediction psychological difficulties.

\section{Participants and Procedure}

Data of 299 mothers $\left(\mathrm{M}_{a g e}=40.2\right.$ years, $\mathrm{SD}_{a g e}=4.79$, age-range $=22-54$ years $)$ and their children $\left(\mathrm{M}_{a g e}=7.96\right.$ years, $\mathrm{SD}_{a g e}=1.36$, Females=139) living in the Italian national territory were collected through an online survey from April $1^{\text {st }}$ to April $9^{\text {th }}, 2020$. Participants were recruited through word of mouths, social media groups, and online advertisements on research-related websites. All participants were at least 18 years old and have children between 6 to 10 years of age. As the first step of the survey, the respondents had to explicitly agree to participate in the study in an online informed consent. Then, they were asked some general questions concerning sociodemographic characteristics including age, gender, educational level, and employment status (i.e., working regularly, stopped working, not working, home working), the number of children, as well as some questions related to the COVID-19 situation (e.g., how much do they feel scared by the COVID-19, where they obtain information about the novel coronavirus, whether they know people infected by or deceased due to COVID-19). Then, mothers were asked to fill the survey thinking 
about their habits, behaviors, and emotions and of those of their children. Importantly, questions were referred at their experiences at present, during the quarantine (from $1^{\text {st }}$ to the $9^{\text {th }}$ of April, after three weeks of confinement), and retrospectively to the week before the national total lockdown (i.e., February $24^{\text {th }}$ to $29^{\text {th }}$ ). The survey took about $30 \mathrm{~min}$ to be completed. The project was approved by the Ethical Committee of Psychological Research of the University of Padova (Prot. $\left.n^{\circ} 3521\right)$.

\section{Measures}

\section{Behavioral factors}

Sleep Quality. Mothers' sleep quality was assessed using the total score of the Pittsburgh Sleep Quality Index (PSQI; Italian version by Curcio et al., 2013), a valid, reliable, and widely used self-report questionnaire. The range of this 18 -item scale is from 0 to 21 , with higher scores indicating worse sleep quality. A total score of $>5$ indicates poor sleep quality. From items 1 and 3 of the same questionnaires, we extracted the average bedtime (at what time they went to bed to sleep, hh:mm) and waketime (at what time they wake up in the morning, hh:mm) of the mothers. From bedtime and waketime, we also derived the sleep midpoint (hh:mm).

Children's sleep quality was assessed using the total score of the Sleep Disturbance Scale for Children (SDSC; Bruni et al., 1996). This questionnaire, validated in Italian children aged 6.515.3 years, is composed of 26 items with a total score ranging from 26 to 130 . Higher scores indicating greater sleep difficulties. A score >39 can be considered a cut-off for identifying children with potential disturbed sleep. Also, we asked the mothers to report the average bedtime, waketime of their children and again we derived the sleep midpoint.

Time perception. We adapted seven items from the Subjective Time Questionnaire (STQ; Italian version by Mioni, Wittmann, Prunetti, \& Stablum, 2020) to tap mothers' subjective feelings of time. The questionnaire contains several parts consisting of questions concerning everyday experiences and a retrospective look at long past time intervals as well as the subjective feeling of 
time, and metaphors of time. To the purpose of the present study, we asked how fast one hour, a day, and a week has passed (referring to the first week of February, and last week), with responses ranging from $1=$ very slow to $5=$ very fast. We also used questions concerning statements that refer to the feeling of time pressure/time compression (two statements, e.g., "I often think that time is running out.") or to the feeling of time expansion/time affluence (2 statements, e.g., "My time is not filled."; anchors: $1=$ strong rejection and 5=strong approval).

For children, we used three items to test the subjective feeling of time and time management. Two out of three items were selected from Porcelli et al. (2018) asking "He/she does not experience that time has passed" and "He/she doesn't respect the routine". The third item investigates children' subjective perception of boredom which is strongly correlated with time (Zakay, 2014): "Is he/she experiencing boredom".

\section{Psychological factors}

The Strengths and Difficulties Questionnaire-Parent version (SDQ-P). The SDQ-P (Goodman, 1997; Italian version validated by Tobia \& Marzocchi, 2018) is a parent-report screening tool aimed to evaluate children's strengths and difficulties as detected by their parents. The SDQ-P included 25 items developed on a 3-point Likert scale ( $0=$ "Not True"; 1 ="Somewhat true"; $2=$ "Certainly true"). The questionnaire is characterized by 5 subscales: Emotional symptoms (EMO, 5 items), Peer problems (PEER, 5 items), Hyperactivity-Inattention (HYPER, 5 items), Conduct problems (COND, 5 items), and Prosocial behaviors (PROS, 5 items) and a Total Difficulty Score (TDS). We collected data only on EMO, HYPER, and COND subscales, and computed the Psychological Difficult Score (PDS) as the sum of the scores on these three subscales. Moreover, from the raw score in each of the subscale, we also calculated the proportion of children at high risk of developing clinical symptoms taking into account age and gender of the children (Tobia \& Marzocchi, 2018). 
The Strengths and Difficulties Questionnaire - 18+ (Goodman, 1997) is a screening selfreport identifying strengths and difficulties in individuals older than 18 years. SDQ 18+ presents the same structure as the parent's version. Again, we collected data only on EMO, HYPER, and COND subscales, and computed the PDS.

The Difficulties in Emotion Regulation (DERS). The DERS (Italian version validated by Giromini, Velotti, de Campora, Bonalume, \& Cesare Zavattini, 2012; Gratz \& Roemer, 2004) is a 36-item questionnaire aimed to detect multiple features of emotion regulation, on a 5-point Likert scale (from 1="almost never" to 5="almost always"). The total score of the DERS was used in the current study.

\section{Statistical analysis}

The $\chi^{2}$ and McNemar's test were used to differences in independent and paired nominal variables, respectively.

For both mothers and children, a series of linear mixed models (LMM) were conducted to assess the change in our main variables of interest, with participants as Random factor, and working condition (regular working, smart working, stopped working, and not working), Lockdown (before the home confinement, during the home confinement), the gender of the children (only for the children's models), as fixed effects, and the DERS total scores and children' age as covariates. Posthoc comparisons were corrected using the Holm method.

To assess the relationship between changes in emotional symptoms and behavioral difficulties and changes in sleep quality and timing, taking into account demographic factors, we built a multiple linear regression model with the change in children's psychological difficulties (i.e., the PDS) as the dependent variable and, as covariates, the changes in sleep quality (SDSC) and timing (sleep midpoint), changes in boredom level, children's age, mothers' fear of COVID-19 contagion, and change in mothers' psychological difficulties (i.e., the PDS). For all the analyses, the level of significance was set at $p<.05$. 


\section{Results}

\section{Descriptive statistics}

The age of the sample was equally distributed between 6 and 10 years old $\left(\chi^{2} 4=5.90\right.$, $p=.207)$, with a similar proportion of females (46.5\%) and males (46.5\%, $\left.\chi^{2} 2=1.47, p=.225\right)$. All the children used to go to school before the restrictions. All but one child were informed by the family about the COVID-19 situation using different modalities, mainly by videos (58.9\%) and through scientific information (45.8\%). For the younger (6-7 years, $n=122)$, the COVID-19 situation was also explained using specifically created fairy-tales (35\% of this sample). Before the lockdown, 31 children $(10.5 \%)$ underwent psychological treatments, whereas during the lockdown the number of children followed by a psychologist was $34(11.3 \%$; 4 new children whereas 1 child stopped the clinical treatment).

Most of the mothers $(74.3 \%)$ were in the $35-45$ years old range. The majority of them $(\mathrm{n}=239,79.9 \%)$ used to work before the lockdown. During the lockdown, only $19.7 \%$ of them continued working outside their home, whereas $47.7 \%$ had to start working from home in smart modality, and $32.7 \%$ had to stop working. Mothers had a relatively high fear of contagion (17.7\% reported a high level of fear, whereas $47.5 \%$ a moderate fear), $28.7 \%$ of them knew someone who had contracted COVID-19 and 8.7\% knew someone who passed away due to the contagion. 
Table 1. Demographics and descriptive information of the mothers and their children for each working condition.

\begin{tabular}{lc} 
MOTHERS & Mean (SD) \\
\hline Age (years) & $40.02(4.79)$ \\
Number of children in the family & $2.0(0.7)$ \\
\hline Area of Italy & $\mathbf{N}(\%)$ \\
North & $231(78 \%)$ \\
Center & $11(3.7 \%)$ \\
South & $54(18.2 \%)$ \\
\hline COVID-19 items & \\
$\begin{array}{l}\text { Do you fear getting infected (0- } \\
\text { 3)? }\end{array}$ & $1.80(0.76)$ \\
\hline $\begin{array}{l}\text { Do you know someone who has } \\
\text { tested positive for COVID-19? }\end{array}$ & $\mathbf{N}(\%)$ \\
Infected & $86(28.8 \%)$ \\
Deceased & $26(8.7 \%)$ \\
\hline CHILDREN & Mean (SD) \\
\hline Age (years) & $7.96(1.36)$ \\
\hline Gender (M) & $\mathbf{N}(\%)$ \\
\hline & $160(53.5)$ \\
\hline
\end{tabular}

\section{Home confinement impact on children}

The LMM on children bedtime showed a significant effect of the Lockdown $\left(\mathrm{F}_{1,291}=651.87\right.$, $p<.001$, Figure 1a), regardless of the mothers' working condition, and a significant covariation with Age $\left(\mathrm{F}_{1,289}=17.48, p<.001\right)$, with older children that went to bed later during the lockdown (coeff. $=0.14, S E=0.03, t=4.18)$. In general, children went to bed about $1 \mathrm{hr}$ and $18 \mathrm{~min}$ later than before the lockdown. Also, waketime significantly changed during lockdown $\left(\mathrm{F}_{1,291}=663.48\right.$, $p<.001$, Figure $1 \mathrm{~b}$ ), with children waking up about $1 \mathrm{hr}$ and $50 \mathrm{~min}$ later than before the lockdown. Overall, the sleep midpoint shifted of $\sim 1 \mathrm{hr}$ and $36 \mathrm{~min}$.

The sleep quality showed a significant effect of the Lockdown $\left(\mathrm{F}_{1,291}=4.93, p=.027\right)$, regardless of the mothers' working condition, and a significant interaction of the 
Gender $\times$ Lockdown $\left(\mathrm{F}_{1,291}=4.38, p=.037\right)$, showing than the worsening in quality was driven by females $(p=.022)$ and not males ( $p>$.99). Age was not a significant covariate, but DERS total score did covariate positively with the change in sleep quality $\left(\mathrm{F}_{1,289}=38.52, p<.001\right.$; coeff. $=1.69$, $S E=1.01, t=6.21)$. The proportion of children with potential disturbed sleep (i.e., SDCS $>39$ ) did not increase significantly $\left(39.5 \%\right.$ to $\left.41.1 \% ; \chi^{2}{ }_{1}=0.37, p=.541\right)$.

Emotional symptoms showed a trend for worsening during lockdown $\left(\mathrm{F}_{1,291}=3.84, p=.051\right.$, Figure 1c), and again the mothers' DERS total score covaried positively with their children's emotional symptoms $\left(\mathrm{F}_{1,289}=14.29, p<.001 ;\right.$ coeff. $\left.=0.02, S E=0.005, t=3.78\right)$. The lockdown significantly increased conduct problems $\left(\mathrm{F}_{1,291}=28.17, p<.001\right.$, Figure 1c $)$, and the DERS total score resulted a significant covariate $\left(\mathrm{F}_{1,289}=14.68, p<.001 ;\right.$ coeff. $\left.=0.02, S E=0.005, t=3.83\right)$. Also hyperactivity-inattention increased during the lockdown $\left(\mathrm{F}_{1,291}=16.31, p<.001\right.$, Figure $\left.1 \mathrm{c}\right)$, and, in general, was higher in males compared to females $\left(\mathrm{F}_{1,291}=7.53, p=.006\right)$, although the interaction Gender $\times$ Lockdown was not significant $\left(\mathrm{F}_{1,291}=0.71, p=.402\right)$. Again, the DERS total score was a significant covariate $\left(\mathrm{F}_{1,289}=8.10, p=.005 ;\right.$ coeff. $\left.=0.01, S E=0.004, t=2.85\right)$. 

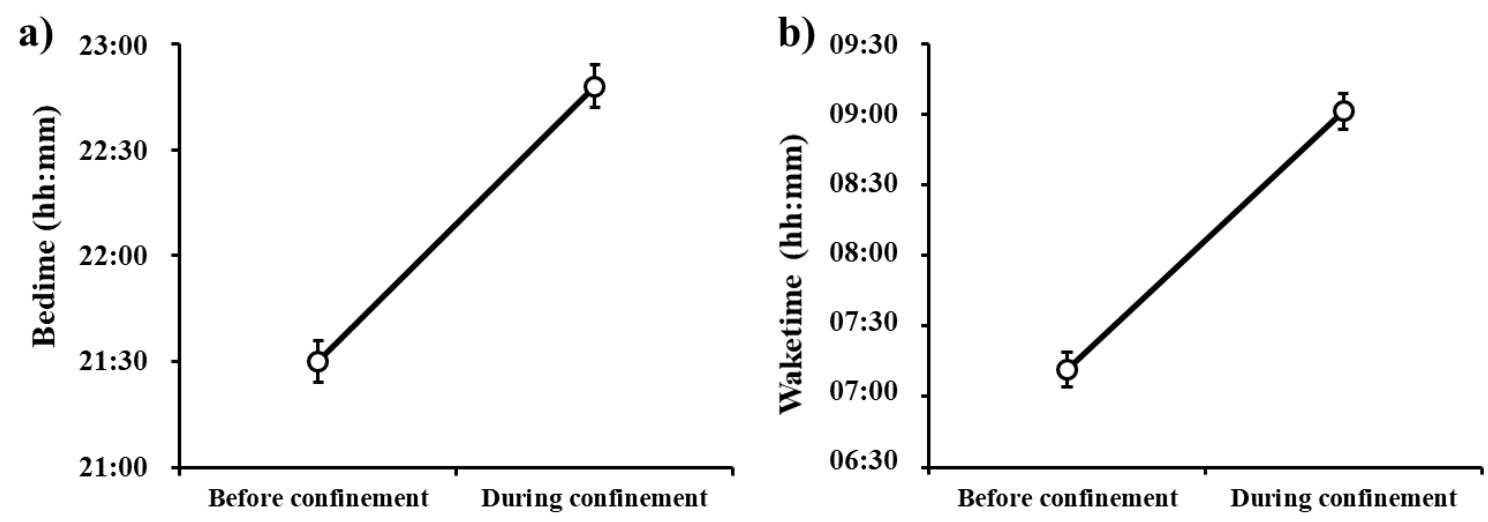

c)

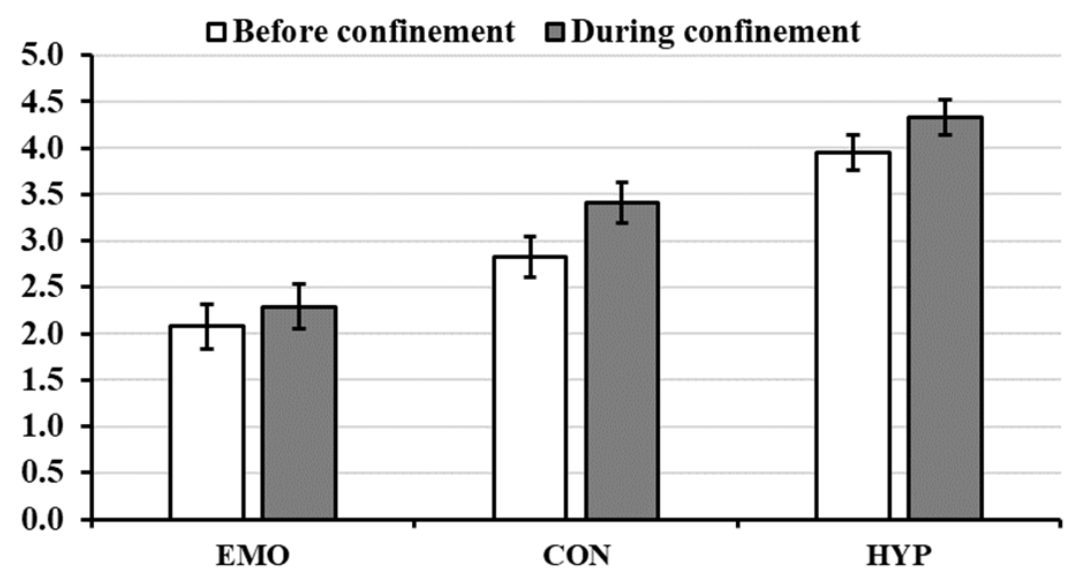

Figure 1. Changes in children's a) bedtime b) waketime, c) SDQ subscales as a function of the presence of the home confinements. For panel EMO: Emotional symptoms subscale; CON: Conduct problems subscale; HYP: Hyperactivity/inattention subscale. Error bars represent 95\% confidence interval.

Regarding time experience, children had more difficulties to keep track of the passage of time during the home confinement $\left(\mathrm{F}_{1,291}=3.80, p=.011\right)$, and they were less able to attend to the daily routines $\left(\mathrm{F}_{1,291}=133.53, p<.001\right)$. As expected, children felt more bored $\left(\mathrm{F}_{1,291}=99.11, p<.001\right)$, and this effect was stronger in males than females (Gender $\times$ Lockdown: $\left.\mathrm{F}_{1,291}=6.11, p=.014\right)$. Also, we observed a significant Gender $\times$ Lockdown $\times$ Work interaction $\left(\left(\mathrm{F}_{1,291}=99.11, p<.001\right)\right.$, showing an increased feeling of boredom in all children (all $p^{\prime} s<.023$ ) except for females with mothers not working or working regularly $(p=.99)$. 


\section{Home confinement impact on mothers}

The LMM on mothers bedtime showed a significant effect of the Period $\left(\mathrm{F}_{1,295}=253.08\right.$, $p<.001)$, and a significant interaction of the Work $\times$ Lockdown $\left(\mathrm{F}_{3,293}=7.31, p<.001\right)$ with mothers who stopped working going to bed $1 \mathrm{hr}$ and 24 min later $(p<.001)$, whose did not working $1 \mathrm{hr}$ and 12 min later $(p<.001)$, the ones starting to work in smart modality 54 min later $(p<.001)$, whereas whose keep working regularly outside their home 36 min later $(p=.003)$. For waketime, we observed only a significant effect of the Lockdown $\left(\mathrm{F}_{1,295}=284.34, p<.001\right)$, and a main effect of Work $\left(\mathrm{F}_{1,293}=3.86, p=.010\right)$, but not a significant interaction of the Work $\times$ Lockdown $\left(\mathrm{F}_{3,295}=1.50\right.$, $p=.215$, Figure $2 \mathrm{a}$ ), although we observed some nominal differences: mothers who stopped working woke up $\sim 1 \mathrm{hr}$ and 17 min later, whose did not work $\sim 1 \mathrm{hr}$ and 27 min later, whose starting to work in smart modality $\sim 1 \mathrm{hr}$ and 16 min later, whereas whose keep working regularly outside their home woke up $\sim 59$ min later (Figure 2b). Also, the DERS total score resulted a significant covariates $\left(\mathrm{F}_{1,293}=4.70, p=.031\right.$, coeff. $\left.=0.006, S E=0.003, t=2.17\right)$. Overall, the sleep midpoint shifted of $\sim 1 \mathrm{hr}$ and $10 \mathrm{~min}$.

Sleep quality decreased during the lockdown $\left(\mathrm{F}_{1,295}=101.42, p<.001\right)$, regardless of the mothers' working conditions. The DERS total score did covariate positively with the change in sleep quality $\left(\mathrm{F}_{1,293}=35.16, p^{<.001}\right.$; coeff. $\left.=0.04, S E=.007, t=5.93\right)$. The proportion of mothers with poor sleep (i.e., PSQI $>5$ ) significantly increased from $19.7 \%$ to $45.8 \%,\left(\chi^{2}{ }_{1}=53.4, p<.001\right)$.

Emotional symptoms got worse during home confinement $\left(\mathrm{F}_{1,295}=8.72, p=.003\right)$. Although we observed a main effect of Work $\left(\mathrm{F}_{3,293}=3.31, p=.021\right)$, with higher emotional symptomatology in mothers who stopped working compared to those in smart working, there was no significant interaction of the Work $\times$ Lockdown $\left(\mathrm{F}_{3,295}=0.58, p=.629\right)$. 


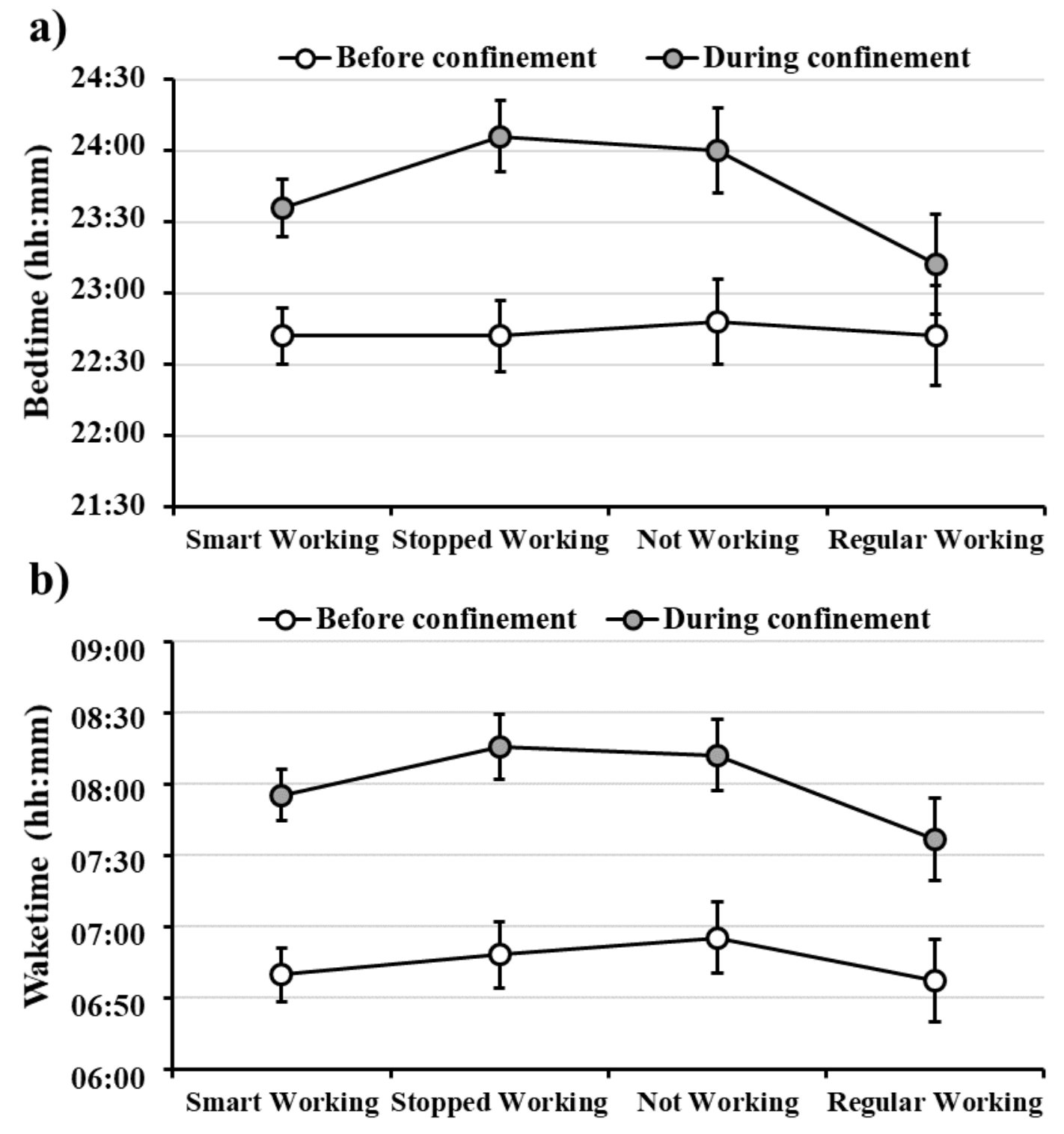

Figure 2. Changes in mothers' a) bedtime, b) waketime, as a function of the presence of the home confinement and the mothers' working condition. Error bars represent 95\% confidence interval.

Regarding time experience, mothers reported that the speed of the hours $\left(\mathrm{F}_{1,295}=42.84\right.$, $p<.001)$, days $\left(\mathrm{F}_{1,295}=46.06, p<.001\right)$, and the week $\left(\mathrm{F}_{1,295}=45.51, p<.001\right)$, were slowing down during the confinement. Interestingly, for both the speed of hours and the week, we observed the stronger effect in mothers who started to work in smart (home) modality and in those who stopped working (all $p$ 's<.001). Also, they felt a marked decrease in time pressure $\left(\mathrm{F}_{1,295}=145.31, p<.001\right)$, and an increase in time expansion $\left(\mathrm{F}_{1,295}=99.20, p<.001\right)$ during the confinement period. 


\section{Predictors of children's emotional symptoms}

The multiple regression models (Table 2) showed that the change in children's emotional symptoms and difficulties was predicted by their sleep quality (i.e., worst sleep quality, worse emotional difficulties), the increasing boredom, and by the mothers' emotional symptoms and difficulties. Interestingly, the change in sleep timing, age of children, and mothers' Fear of COVID19 were not significant predictors of the change in children's psychological difficulties. Also note that testing other models including other COVID-19 related variables (e.g., knowing someone who was infected or died as a consequence of COVID-19) did not improve the presented model.

Table 2. Multiple regressions of changes in the Psychological Difficulties Score (PDS) in children

\begin{tabular}{lcccc} 
& \multicolumn{4}{c}{$\Delta$ Psychological Difficulties Score } \\
\cline { 2 - 5 } & $b(95 \% \mathrm{CI})$ & std. $\beta$ & $t$ & $P$ \\
\hline Intercept & $0.31(-2.10,2.72)$ & 0 & 0.25 & .802 \\
Age & $-0.13(-0.40,0.13)$ & -0.05 & -0.96 & .338 \\
A Sleep Quality & $\mathbf{0 . 1 4}(\mathbf{0 . 1 0 , 0 . 1 8})$ & $\mathbf{0 . 3 4}$ & $\mathbf{6 . 8 7}$ & $<.001$ \\
A Sleep Timing & $0.20(-0.21,0.06)$ & 0.05 & 0.96 & .337 \\
A Boredom & $\mathbf{0 . 9 4}(\mathbf{0 . 5 3}, \mathbf{1 . 3 5})$ & $\mathbf{0 . 2 3}$ & $\mathbf{4 . 5 3}$ & $<.001$ \\
Mothers' APDS & $\mathbf{0 . 2 3}(\mathbf{0 . 1 3}, \mathbf{0 . 3 3})$ & $\mathbf{0 . 2 3}$ & $\mathbf{4 . 6 7}$ & $<.001$ \\
Mothers' Fear of COVID-19 & $0.39(-0.08,0.87)$ & 0.07 & 1.62 & .106 \\
\hline Model Fit & $F(6,292)=23.1$ & & & \\
Adj. $R^{2}$ & $p<.001$ & & & \\
\hline
\end{tabular}

Note. $b=$ unstandardized beta; std. $\beta=$ standardized beta; $C I=$ confidence intervals. $\Delta$ Sleep Quality: change in Sleep Disturbance Scale for Children. $\Delta$ Sleep Timing: change in sleep midpoint. Mothers $\Delta$ PDS: Psychological Difficulties Score in mothers.

\section{Discussion}

The present study aimed at evaluating the effect of prolonged home confinement during the Italian lockdown in school-age children and their mothers in terms of daily routines, sleep quality and timing, and their associations with psychological difficulties. Although it is well-known that the disruption of children' basic routines might be detrimental for preserving psychosocial and physical well-being (Brazendale, et al., 2017), to date there is a lack of empirical studies focusing on this topic in pandemic scenarios. 
Here, as expected, mothers reported significant changes in their children' sleep habits, with later bedtime and waketime, especially for older children, compared to the pre-lockdown period. Moreover, they were less prone to respect the daily routines and to keep track of the passage of time. We also observe increased emotional, conduct, and hyperactive symptoms, and the increase in these psychological difficulties was predicted by the change in sleep quality, boredom, and mothers' psychological difficulties.

This data are in line with our previous study in Italian pre-schooler (Di Giorgio, et al., 2020), where we observed a shift in sleep timing, although it was less delayed than school-age children since the latter are likely to be more frequently absorbed in activities before going to sleep (e.g., tv, videogames, reading). Moreover, the present study highlighted a worsening of emotional symptoms in children during the lockdown, due to the impact of changes in daily habits (e.g. sleep quality), restrictions imposed by pandemic (e.g. boredom), and a higher amount of their mothers' psychological distress. Presumably, school-age children psychological well-being seemed to be affected more by fatigue in adjustment to prolonged home confinement and to a reduction of in peer-relationships in respect of factors specifically related to COVID-19 (e.g. fears to be infected by the novel coronavirus). Interestingly, although children showed a mild worsening in sleep quality, the rate of potential disturbed sleep did not increase significantly, as we observed in pre-schoolers (Di Giorgio et al., 2020). The potential risk for incurring in poor sleep due to the home confinement may have been attenuated by the later sleep timing, more aligned with the biological clock of older/pre-puberal children (Carskadon \& Barker, 2020). Moreover, the change in children' sleep quality was associated with their mothers' difficulties in regulating their emotions. This results can be interpreted in line with the idea that emotional weaknesses in mothers are often associated with sleep difficulties in children (Stoléru, Nottelmann, Belmont, \& Ronsaville, 1997), but also as a response bias: mothers with higher emotional weaknesses identify their children as more problematic and symptomatic. Nevertheless, this small change in sleep quality was predictive of the 
increase in their psychological difficulties, in line with the idea that poor sleep can "set the stage for behavioral dysregulation and risks for psychopathology" (Carskadon \& Barker, 2020, p.2).

Indeed the observed increase in psychological difficulties during the lockdown indicates that school-age children are at risk of developing internalized symptoms - such as sadness or concern and externalized symptoms - such as irritability or difficulties in concentrating. The increase in psychological difficulties is in line with the few reports showing that restrictions on mobility imposed by the novel coronavirus outbreak impacted negatively on children's daily routines, psychological well-being (anxiety, frustration) and boredom, mainly connected to unexpected and prolonged school closure (Jiao, et al., 2020). Indeed, home confinement and school closure resulted in the interruption of in-person relationships with peers, as well as the reduction of personal space at home (Wang, et al., 2020).

Similarly to what we reported previously (Di Giorgio et al., 2020), also in mothers, we observed a significant bed and waketime postponement, a worsening of sleep quality during the lockdown, and an increment of psychological symptoms, consistent with other recent paper on Italian (Casagrande et al., 2020; Cellini et al., 2020; Gualano et al., 2020) and other adult samples from several different countries (Beck et al., 2020; Blume et al., 2020; Lin et al., 2020; Voitsidis et al., 2020; Wright et al., 2020). Importantly, mothers' working conditions seem to play a crucial role in keeping or disrupting healthy habits in a pandemic scenario. Mothers who kept working regularly outside their home during the lockdown reported a more regular sleep pattern, whereas mothers who stopped working showed more emotional symptoms and relevant changes in their time perception. This is also consistent with our previous data in preschoolers (Di Giorgio et al., 2020).

Our data should be interpreted in light of some limitations. First, the current study is crosssectional and data about children were collected only by mothers'reports. Although literature supported this method, the distress scenario in which mothers were involved might affect their perception of children well-being. Then, modest sample size and recruitment biases did not allow to generalize results. The online survey could reach only mothers prone to the use of electronic 
devices, but in times of social distancing online recruitment represented the only way accessible. Further, no information about family support for mothers (e.g partner, grandparents), which could have mitigated the impact quarantine burden, was collected. Finally, the present research employed retrospective questions in order to compare the present situation to the previous baseline before the lockdown. Although this method has some pitfalls and biases, however it could be considered reliable (Hipp, Bünning, Munne, \& Sauermann, 2020).

Overall, given the evidence of the adverse behavioural and psychological impact of home confinement and social restrictions, effective measures are needed to be in place to mitigate their long-term effects on children and their mothers, especially those in the smart working condition. First, managing sleep problems as best as possible during home confinement can limit stress and possibly prevent behavioural and emotional problems (Altena et al., 2020; Crew et al., 2020). Second, based on the present and other studies in literature, the Government should implement both prevention and intervention programs for increasing psychological well-being in children and their caregivers' in the event of a new COVID-19 wave that may occur during the cold seasons (i.e., fall and winter) or in case of other epidemic situations. Also, specific projects to sustain working mothers in handling their children's needs are urgently warranted. Finally, policymakers should implement modeling and observational studies to guide the opening of schools once the pandemic is under control. This is the crucial point in Italy right now, where the Government has established a task force involved to outline the guidelines for safe schools reopening in September 2020, but can be extended to several countries experiencing partial and total lockdown due to the pandemic situation. 


\section{References}

Altena, E., Baglioni, C., Espie, C. A., Ellis, J., Gavriloff, D., Holzinger, B., . . Riemann, D. (2020). Dealing with sleep problems during home confinement due to the COVID-19 outbreak: practical recommendations from a task force of the European CBT-I Academy. Journal of Sleep Research.

Baum, K. T., Desai, A., Field, J., Miller, L. E., Rausch, J., \& Beebe, D. W. (2014). Sleep restriction worsens mood and emotion regulation in adolescents. Journal of Child Psychology and Psychiatry, 55(2), 180-190.

Beck, F., Léger, D., Fressard, L., Peretti-Watel, P., Verger, P., \& Group, C. (2020). Covid-19 health crisis and lockdown associated with high level of sleep complaints and hypnotic uptake at the population level. Journal of Sleep Research, e13119.

Becker, S. P., \& Gregory, A. M. (2020). Editorial Perspective: Perils and promise for child and adolescent sleep and associated psychopathology during the COVID-19 pandemic. Journal of Child Psychology and Psychiatry.

Blume, C., Schmidt, M. H., \& Cajochen, C. (2020). Effects of the COVID-19 lockdown on human sleep and rest-activity rhythms. Current Biology.

Brazendale, K., Beets, M. W., Weaver, R. G., Pate, R. R., Turner-McGrievy, G. M., Kaczynski, A. T., . . von Hippel, P. T. (2017). Understanding differences between summer vs. school obesogenic behaviors of children: the structured days hypothesis. International Journal of Behavioral Nutrition and Physical Activity, 14(1), 100.

Brooks, S. K., Webster, R. K., Smith, L. E., Woodland, L., Wessely, S., Greenberg, N., \& Rubin, G. J. (2020). The psychological impact of quarantine and how to reduce it: rapid review of the evidence. The Lancet.

Bruni, O., Ottaviano, S., Guidetti, V., Romoli, M., Innocenzi, M., Cortesi, F., \& Giannotti, F. (1996). The Sleep Disturbance Scale for Children (SDSC) Construct ion and validation of an instrument to evaluate sleep disturbances in childhood and adolescence. Journal of Sleep Research, $5(4), 251-261$.

Carskadon, M. A., \& Barker, D. H. (2020). Editorial Perspective: Adolescents' fragile sleepshining light on a time of risk to mental health. Journal of Child Psychology and Psychiatry.

Casagrande, M., Favieri, F., Tambelli, R., \& Forte, G. (2020). The enemy who sealed the world: Effects quarantine due to the COVID-19 on sleep quality, anxiety, and psychological distress in the Italian population. Sleep Medicine.

Cellini, N., Canale, N., Mioni, G., \& Costa, S. (2020). Changes in sleep pattern, sense of time and digital media use during COVID-19 lockdown in Italy. Journal of Sleep Research, e13074.

Crew, E. C., Baron, K. G., Grandner, M. A., Ievers-Landis, C. E., McCrae, C. S., Nadorff, M. R., . . . Hansen, K. (2020). The Society of Behavioral Sleep Medicine (SBSM) COVID-19 Task Force: Objectives and Summary Recommendations for Managing Sleep during a Pandemic. Behavioral Sleep Medicine, 1-3. 
Curcio, G., Tempesta, D., Scarlata, S., Marzano, C., Moroni, F., Rossini, P. M., . . De Gennaro, L. (2013). Validity of the Italian version of the Pittsburgh sleep quality index (PSQI). Neurological Sciences, 34(4), 511-519. doi: 10.1007/s10072-012-1085-y

Di Giorgio, E., Di Riso, D., Mioni, G., \& Cellini, N. (2020). The interplay between mothers' and children behavioral and psychological factors during COVID-19: An Italian study.

Giromini, L., Velotti, P., de Campora, G., Bonalume, L., \& Cesare Zavattini, G. (2012). Cultural adaptation of the difficulties in emotion regulation scale: Reliability and validity of an Italian version. Journal of Clinical Psychology, 68(9), 989-1007.

Goodman, R. (1997). The Strengths and Difficulties Questionnaire: a research note. Journal of Child Psychology and Psychiatry, 38(5), 581-586.

Gratz, K. L., \& Roemer, L. (2004). Multidimensional assessment of emotion regulation and dysregulation: Development, factor structure, and initial validation of the difficulties in emotion regulation scale. Journal of Psychopathology and Behavioral Assessment, 26(1), 41-54.

Gregory, A. M., \& O'Connor, T. G. (2002). Sleep problems in childhood: a longitudinal study of developmental change and association with behavioral problems. Journal of the American Academy of Child and Adolescent Psychiatry, 41(8), 964-971.

Gualano, M., Lo Moro, G., Voglino, G., Bert F, \& Siliquini, R. (2020). Effects of Covid-19 Lockdown on Mental Health and Sleep Disturbances in Italy. International Journal of Environmental Research and Public Health, 17(13), 4779.

Guan, H., Okely, A. D., Aguilar-Farias, N., del Pozo Cruz, B., Draper, C. E., El Hamdouchi, A., . . Kontsevaya, A. (2020). Promoting healthy movement behaviours among children during the COVID-19 pandemic. The Lancet Child \& Adolescent Health, 4(6), 416-418.

Hipp, L., Bünning, M., Munne,s S., \& Sauermann, A. (2020). Problems and pitfalls of retrospective survey questions in COVID-19 studies. Survey in Research Methods, 14, 109-114.

Jiao, W. Y., Wang, L. N., Liu, J., Fang, S. F., Jiao, F. Y., Pettoello-Mantovani, M., \& Somekh, E. (2020). Behavioral and emotional disorders in children during the COVID-19 epidemic. The journal of Pediatrics, 221, 264.

Kelvin, A. A., \& Halperin, S. (2020). COVID-19 in children: the link in the transmission chain. The Lancet Infectious Diseases.

Kokou-Kpolou, C. K., Megalakaki, O., Laimou, D., \& Kousouri, M. (2020). Insomnia during COVID-19 pandemic and lockdown: Prevalence, severity, and associated risk factors in France population. Psychiatry Research, 113128.

Lin, L.-y., Wang, J., Ou-yang, X.-y., Miao, Q., Chen, R., Liang, F.-X., . . Wang, T. (2020). The immediate impact of the 2019 novel coronavirus (COVID-19) outbreak on subjective sleep status. Sleep Medicine.

Mioni, G., Wittmann, M., Prunetti, E., \& Stablum, F. (2020). Time perspective and the subjective passage of time in patients with borderline personality disorders. Timing \& Time Perception, 8(1), 86-101. 
Orgilés, M., Morales, A., Delvecchio, E., Mazzeschi, C., \& Espada, J. P. (2020). Immediate psychological effects of the COVID-19 quarantine in youth from Italy and Spain. PsyArXiv. https://doi.org/10.31234/osf.io/5bpfz

Paavonen, E. J., Porkka-Heiskanen, T., \& Lahikainen, A. R. (2009). Sleep quality, duration and behavioral symptoms among 5-6-year-old children. European Child and Adolescent Psychiatry, 18(12), 747.

Pietrobelli, A., Pecoraro, L., Ferruzzi, A., Heo, M., Faith, M., Zoller, T., . . Heymsfield, S. B. (2020). Effects of COVID-19 lockdown on lifestyle behaviors in children with obesity living in Verona, Italy: a longitudinal study. Obesity.

Sprang, G., \& Silman, M. (2013). Posttraumatic stress disorder in parents and youth after healthrelated disasters. Disaster Medicine and Public Health Preparedness, 7(1), 105-110.

Stoléru, S., Nottelmann, E. D., Belmont, B., \& Ronsaville, D. (1997). Sleep problems in children of affectively ill mothers. Journal of Child Psychology and Psychiatry, 38(7), 831-841.

Tobia, V., \& Marzocchi, G. M. (2018). The strengths and difficulties questionnaire-parents for Italian school-aged children: psychometric properties and norms. Child Psychiatry and Human Development, 49(1), 1-8.

Voitsidis, P., Gliatas, I., Bairachtari, V., Papadopoulou, K., Papageorgiou, G., Parlapani, E., .. . Diakogiannis, I. (2020). Insomnia during the COVID-19 pandemic in a Greek population. Psychiatry Research, 113076.

Wang, C., Pan, R., Wan, X., Tan, Y., Xu, L., Ho, C. S., \& Ho, R. C. (2020). Immediate psychological responses and associated factors during the initial stage of the 2019 coronavirus disease (COVID-19) epidemic among the general population in china. International Journal of Environmental Research and Public Health, 17(5), 1729.

Wright, K. P., Linton, S. K., Withrow, D., Casiraghi, L., Lanza, S. M., de la Iglesia, H., .. . Depner, C. M. (2020). Sleep in University Students Prior to and During COVID-19 Stay-at-Home Orders. Current Biology.

Zakay, D. (2014). Psychological time as information: The case of boredom. Frontiers in Psychology, 5, 917. 